\title{
QUANTUM SPACE AND DARK ENERGY
}

\author{
Carlos A. Melendres \\ The SHD Institute \\ 216 F Street, Davis, CA 95616, U.S.A. \\ Email: camelendres@SHDInstitute.org \\ Tel: +1 5304053035
}

May 2017

Keywords: quantum space, expansion of the universe, dark energy; thermodynamics, dark matter, plasma, cosmic epoch, recombination, Cosmological Constant, Quintessence 


\title{
QUANTUM SPACE AND DARK ENERGY
}

\author{
Carlos A. Melendres
}

\begin{abstract}
The accelerated expansion of the universe is of great scientific interest. It is attributed to Dark Energy. We present a quantum theory of space, and a thermodynamic approach to modeling the evolution of the universe, that explain it. Space is a dynamical entity made up of energy quanta. From wave particle duality, they can also be considered as a gas. The universe evolved starting from a point size volume of gas at very high temperature and pressure. Upon expansion and cooling, phase transitions occured resulting in the formation of fundamental particles, radiation, and matter; these nucleate and grow into stars, galaxies, and clusters. From a phase diagram of cosmic composition, we obtained a correlation between dark energy and the energy of space. A repulsive space force causes the expansion of the universe; the space quanta arise from a space field. Using the Friedmann equations, data on the composition of the universe at $3.0 \times 10^{5}\left(\mathrm{a}=5.25 \times 10^{-2}\right)$ years and at present $(\mathrm{a}=1)$, obtained from WMAP studies, are well fitted by our model with an equation of state parameter, $w=-0.7$. The accelerated expansion of the universe, starting at about 7 billion years, determined by BOSS measurements, correlates well with the dominance of dark energy at $7.25 \times 10^{9}$ years $(a=0.65)$. The expansion is attributed to Quintessence.
\end{abstract}




\section{INTRODUCTION}

A question of great scientific interest is why the expansion of the universe is accelerating. It has been attributed to a mysterious "dark energy", whose nature is unknown. There is frantic search for exotic particles to explain it. Some believe that it is a property of space itself $[1,2]$. In essence, the universe consists of matter and energy in a volume of space; its expansion is that of space with attendant dilution of the components. The nature of space has been the subject of debate among philosophers, scientists, and mathematicians from Plato to Descartes, Kant, Newton, Leibniz, and others [3]. It is generally viewed like a canvas where nature's landscape and events are portrayed; it is then treated geometrically and mathematically as a surface in 4dimensional space-time. A physical description is lacking. Here we propose an alternative model of space that considers it as a quantized dynamical entity which is an active participant in the evolution of the universe and one of its major components.. We show that the model provides a mechanism to explain the accelerated expansion of the universe and elucidate the nature of dark energy.

\section{A MODEL OF SPACE AND COSMIC EVOLUTION}

It has been thought of that space is not truly empty, that vacuum contains virtual particles that pop in and out of existence, and used to explain the Casimir effect [4]. It seems reasonable to take a view that space contains energy and is quantized. In our model, we consider space to consist of quanta that propagate as waves and can be described by the Planck quantum energy expression:

$$
\mathrm{E}=\mathrm{hc} / \lambda
$$

The symbols have their usual meaning: $E$, is the energy; $\lambda$, the wavelength, and $h$, the Planck constant. The expression defines the equivalence of space and energy, in analogy to the relation between energy and mass, given by Einstein's equation, $E=\mathrm{mc}^{2}$. Thus, energy is inherent in space and space in energy. We shall call the quantal units of space as "spaceons". The space waves (spaceons) can be thought of as a carrier of energy and the thread that weaves the fabric that contains the universe. From the principle of wave- particle duality, the spaceons can also be thought of as a gas which obeys the equation of state [5],

$$
\mathrm{PV}=\mathrm{N}_{0} \mathrm{~K}_{\beta} \mathrm{T} \text {. }
$$

$\mathrm{P}$ is the pressure of the gas, $\mathrm{V}$ the volume, $\mathrm{T}$ the temperature, $\mathrm{N}$ the Avogadro and $\mathrm{K}_{\beta}$ the Boltzman constant.

With this theory we can model the gross features of the evolution of the universe as follows: The universe started (at time, $t=0$ ) as a very small volume of compressed gas (the spaceons) at an extremely high pressure and temperature. For example, an Avogadro number of gas particles occupying a volume of $4.2 \times 10^{-33} \mathrm{~m}^{3}$ (a sphere of $0.1 \mathrm{~A}^{0}$ radius), at a pressure of $1.976 \times 10^{65} \mathrm{~Pa}$ will be at conditions during the Planck epoch with a temperature of $10^{32} \mathrm{~K}$. From this initial state, the gas then expands and cools. As it cools to the appropriate temperature phase transition occurs resulting in the formation of fundamental particles, radiation, nuclei, and atoms. Gravitation causes the formation of galaxies and stars; their clumping results in clusters, local groups and superclusters. Details of the events and transformations involved at the various epochs of the evolution of the universe are similar to those of the Big Bang model and are well documented in books on Cosmology $[6,7,8]$. They need not be repeated here. The universe with radiation, matter, and spaceons continues to expand as we know it.

Our model differs from that of the Big Bang; it is simpler. It avoids the problem of singularity. The creation of the universe did not start with a "bang". The processes of expansion 
of spaceons and their phase transformation (nucleation and growth) to form matter occured in an orderly fashion, following well known physico-chemical processes (This is analogous to the formation of snow and ice, on a cold winter day). A cosmic inflation stage is not necessary to form a universe which is isotropic and homogeneous.

\section{RESULTS AND DISCUSSION}

\subsection{Composition of the Universe}

Several methods are used to determine the composition of the universe. Results of the WMAP satellite studies [9] gave the composition shown in Table 1 soon after the Big Bang and at present:

Table 1-Composition of the Universe

\begin{tabular}{lcc}
\multicolumn{2}{c}{ At the Big Bang } & At Present (13.8 billion years after) \\
Dark Matter & $63 \%$ & $24 \%$ \\
Dark Energy & & $71.4 \%$ \\
Ordinary Matter & $\overline{12} \%$ & $4.6 \%$ \\
Neutrinos & $10 \%$ & - \\
Photons & $15 \%$ & -
\end{tabular}

The composition during the cosmic evolution is also well documented $[6,7,8]$.Table 2 shows this as a function of pressure $(\mathrm{P})$ and temperature $(\mathrm{T})$ at various times and Cosmic Epochs.

Table 2.-Composition of the Universe during its Evolution

Time (after Big Bang)/ Temperature (K) Pressure (Pa) Composition (Cosmic Epoch)

$\begin{array}{lccc}0 \text { to } 0.02 \mathrm{sec} / & 10^{11}-10^{9} & 10^{32}-10^{27} & \begin{array}{c}\text { I. Fundamental particles } \\ \text { + radiation }\end{array} \\ \begin{array}{l}\text { (Quark,Hadron,Lepton, } \\ \text { Electrons,Protons,Neutrons) }\end{array} & & & \text { P }\end{array}$

0.02 to $300 \mathrm{sec} /$

(Nucleosynthesis)

$300 \mathrm{sec}$ to $3.8 \times 10^{5} \mathrm{yrs} /$

(Photon to Recombination)

$3.8 \times 10^{5}$ to $10^{9} \mathrm{yrs} /$

(Dark Ages to Matter

dominated)

$10^{9}$ to $13.8 \times 10^{9} \mathrm{yrs} /$

(Dark Energy dominated)

$$
10^{9}-10^{8} \quad 10^{27}-10^{15}
$$

$$
10^{8}-10^{3}
$$

$$
10^{15}-10^{-11}
$$

$3 \times 10^{3}-4$

$$
10^{-11}-10^{-22}
$$

$4-2.7$
II. Nuclei of $\mathrm{H}, \mathrm{He}, \mathrm{D}, \mathrm{Li}$ + radiation

III. Plasma of ionized $\mathrm{H}$, He and e-

IV.Matter in galaxies,stars space, planets-gases, plasma, solid

V: Dark Energy

At temperatures above $10^{11} \mathrm{~K}$, the universe was a primeval hot gas from which fundamental particles (quarks, leptons, hadrons, protons, neutrons, electrons) and radiation were created. Upon cooling to about $10^{9} \mathrm{~K}$, nucleosynthesis occurred to produce nuclei of $\mathrm{H}, \mathrm{He} \mathrm{Li}$ 
and D. At about $10^{8} \mathrm{~K}$ a plasma phase was formed which consisted of electrons and positive ions of $\mathrm{H}$ and He. Radiation (photons, neutrinos) was ever present and dominated the early epoch of the universe. Further cooling of the plasma until about $3 \times 10^{3} \mathrm{~K}$ resulted in recombination of electrons with positive ions of $\mathrm{H}$ and $\mathrm{He}$, converting the plasma to gases. As the universe continued to expand and cool, matter continued to form which, through the action of gravity, became galaxies, stars, and clusters. The universe cooled to $2.7 \mathrm{~K}$ as indicated by the Cosmic Microwave background. The primary constituents of the universe are: gases $(\mathrm{H}, \mathrm{He})$, plasma of electrons, protons, and He ions, ordinary matter (gasses, solids, dust, in stars, galaxies, clusters, intergalactic space) and spaceons ( gas at all temperatures); radiation is ever present.

Thermodynamics is a very powerful method for obtaining compositional information [10]. From data on composition as a function of time (cooling curves) one can construct a phase diagram (similar to the well known water phase diagrams [5] with gas, liquid, solid $\mathrm{H}_{2} \mathrm{O}$ of different crystalline forms). While such diagrams are constructed normally for the components of matter, the equivalence of matter and energy, allows one to do the same for the universe as a system. Dark energy and dark matter are components of the universe. We have constructed a thermodynamic phase diagram $[5,10]$ for our model universe with spaceons using the data in Table 2.

The result is shown in Fig.1. (Note that the figure is schematic and the temperature is not drawn to scale in order to highlight the phases formed). The major phases are: matter (IV, solid,

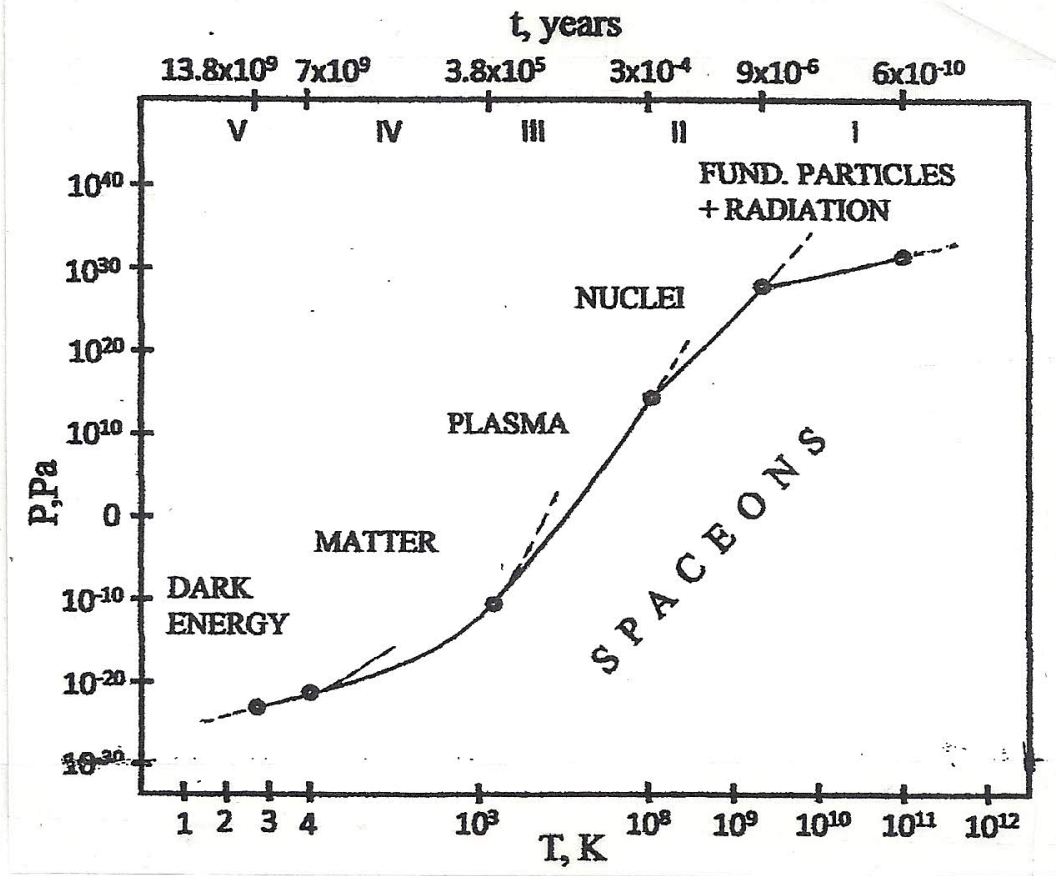

Fig. 1- Phase Diagram of the Universe with Spaceons

gasses, plasma formed in galaxies, stars, clusters), a plasma phase (III) formed immediately after nucleosynthesis, dark energy (V) and spaceons (gas). The broken lines indicate the overlap between various phases during the process of formation. Dark matter is not indicated since its nature remains unknown. All phases are in contact with spaceons at all epochs of cosmic evolution. This is as it should be since space is in contact with all elements of the universe at all times. At extremely high temperatures and pressures (right end), fundamental particles and 
radiation are indistinguishable from the hot spaceons; while at low temperature/pressure (left end), dark energy overlaps with the cold spaceons. These end points are called critical points, where the 2 phases co-exist. In the case of water, for example, at the critical temperature of 647 $\mathrm{K}$ and pressure of $2.2064 \times 10^{7} \mathrm{~Pa}$, liquid water is indistinguisbale from its vapor phase.

\subsection{Dark Energy and the Expansion of the Universe}

Present understanding of Dark Energy is poor to the extent that it is dubbed as something mysterious $[1,2,11,12]$. It is hypothesized to be an unknown form of energy that permeates all of space uniformly. It is invisible and difficult to study because it does not interact with radiation, hence cannot be investigated spectroscopically. It only interacts with gravity. Its density is very low, less than ordinary or dark matter, it converts to dark matter, was less in the past than at present, and it functions as anti-gravity. It is believed to be a property of space and causes the accelerated expansion of the universe $[1,2,11,12]$.

Based on our model, the expansion of the universe could be thought of as the propagation of spaceons driven by the pressure and temperature differential of the hot and cold gas states. This corresponds to a transition of the spaceons from a high energy state, i.e.,short $\lambda_{\mathrm{s}}$ ( in eq.1), (small space) to a low energy state, long $\lambda_{\mathrm{s}}$, (large space). Space waves inherently needs to expand. This is a property of space that is anti-gravity, i.e. the pressure of space opposes the force of gravity. From the standpoint of Quantum Field Theory [13], the expansion can be viewed as arising from a force that is associated with a field. The latter is the space field from which emanate space quanta (analogous to the gravitational field with its associated gravitons). It is a scalar field, dubbed "Quintessence", and has been theorized to be the substance that comprises Dark Energy [11].

One can see from our thermodynamic phase diagram (Fig.1) that dark energy is a phase that overlaps with a new entity that we have introduced as a component of the universe, i.e., the spaceons; the two phases are indistinguishable. Hence, it is logical to identify dark energy as the energy of space. The amount of dark energy soon after the Big Bang was relatively small (Table 1) because most of the energy of space has been converted to electromagnetic radiation, dark matter and ordinary matter. Its density remains low as the universe expands.

Our model universe with spaceons is well suited for mathematical treatment using the Friedmann-Lemaitre-Robertson-Walker (FLRW) metric [12] .The second Friedmann equation $[10,11]$ delineates the contributions of the various components of the universe (matter, radiation, and vacuum) to the acceleration of expansion of the universe, i.e.,

$$
\mathrm{d}^{2} \mathrm{a} / \mathrm{dt} \mathrm{t}^{2}=-(8 \pi \mathrm{Ga} / 3)\left\{(1 / 2) \rho_{\mathrm{m}}+\rho_{\mathrm{r}}-N / 8 \pi \mathrm{G}\right\}
$$

We have left out the curvature term normally appearing in 3 ), assuming that the geometry of the universe is flat $(\mathrm{k}=0)$, which seems to be the case at the present time. In the above equation, $a$ is the scale parameter, $G$ the gravitational constant; $\rho_{\mathrm{m}}$ is the mass-energy density of matter, $\rho_{\mathrm{r}}$ that of radiation, and $\Lambda$, the cosmological constant which represents the energy of the vacuum [10]. Based on our model of space, the term "vacuum energy" has lost its meaning and it is preferable to use the term "energy of space". The cosmological constant will then be replaced by the energy density of space, i.e.

$$
\mathrm{d}^{2} \mathrm{a} / \mathrm{dt} \mathrm{t}^{2}=-(8 / 3) \pi \mathrm{Ga}\left\{(1 / 2) \rho_{\mathrm{m}}+\rho_{\mathrm{r}}+\left(1+3 \mathrm{w}_{\mathrm{s}}\right) \rho_{\mathrm{s}}\right\}
$$

$\rho_{\mathrm{S}}$ is the energy density of space and is related to the pressure $P_{S}$ via the equation of state $P_{S}=w_{S}$ $\rho_{\mathrm{s}} ; \mathrm{w}_{\mathrm{s}}$ is the equation of state parameter. A negative pressure would give rise to a positive term for the contribution of $\rho_{s}$ that can cause an acceleration of the expansion.

For the purpose of fitting the observed data (Table 1), it is preferable to use another 
form of the Friedmnan equation. It is common to utilize one involving the Hubble constant, $\mathrm{H}$, which is measured in experiments and to use velocity, da/dt, rather than acceleration $[11,12,13]$. Thus,

$$
a H^{2}=(d a / d t)^{2} a^{2}=\left(H_{0}\right)^{2}\left[\Omega_{m} a^{-3}+\Omega_{r} a^{-4}+\Omega_{s} a^{-3(1+w)}\right]
$$

where: $H$ is the Hubble constant, $H_{0}$ its value at the present time; $\Omega_{i}$ is the density parameter for component $i$ and is given by the quantity $\Omega_{i}=\varrho_{i} / \varrho_{c} ; \varrho_{i}$ is the energy density of $i$ and $\varrho_{c}$ is the critical energy density.

A plot of fractional energy density as a function of of "a" can be constructed to fit the measured composition of the universe at about the time of the Big Bang and at the present time (Table 1).

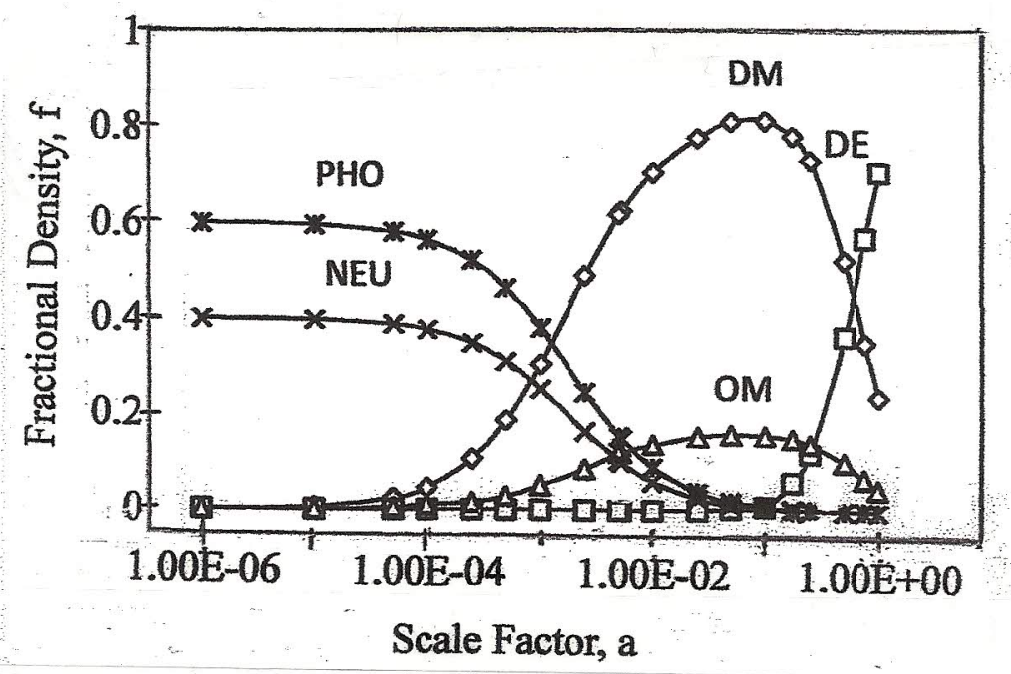

Fig.2- Fractional Energy Density (f) vs Scale Factor (a) DE-Dark Energy; DM-Dark Matter; OM-Ordinary Matter; PHO-Photons; NEU-Neutrinos

The plot in Fig. 2 shows the evolution of the composition of the universe. The dominance of radiation, matter, and dark energy at various times are amply illustrated. As can be seen there is good fit of the data summarized in Table 1 at time $t=13.8$ billion years $(a=1)$ and at the time of the Big Bang, at about $t=380,000$ years ( $a=5.25 \mathrm{E}-02)$, with an equation of state parameter, $\mathrm{w}_{\mathrm{s}}=-0.7$. Dark Energy is very small in the early universe as most of the energy is used in the creation of radiation and particles. As the universe expands, however, the energy densities of radiation and matter continue to dilute and are eventually overcome by the energy density of Dark Energy. This occurs at around 7 billion years at which time the expansion of the universe starts to accelerate. This result has been established by baryon acoustic oscillation measurements in the BOSS project [14]. Fig.3 lends support to these findings. One sees that the energy density of Dark Energy crosses that of the total energy of matter (shown by the the broken line) at $\mathrm{a}=0.65\left(\mathrm{t}=7.25 \times 10^{9}\right.$ years $)$.

Siegel [15] has obtained a similar result using a cosmological constant. However, said approach leads to even more perflexing questions, e.g., why the energy density of dark energy should be constant. Moreover, it does not shed light on its nature and origin. 


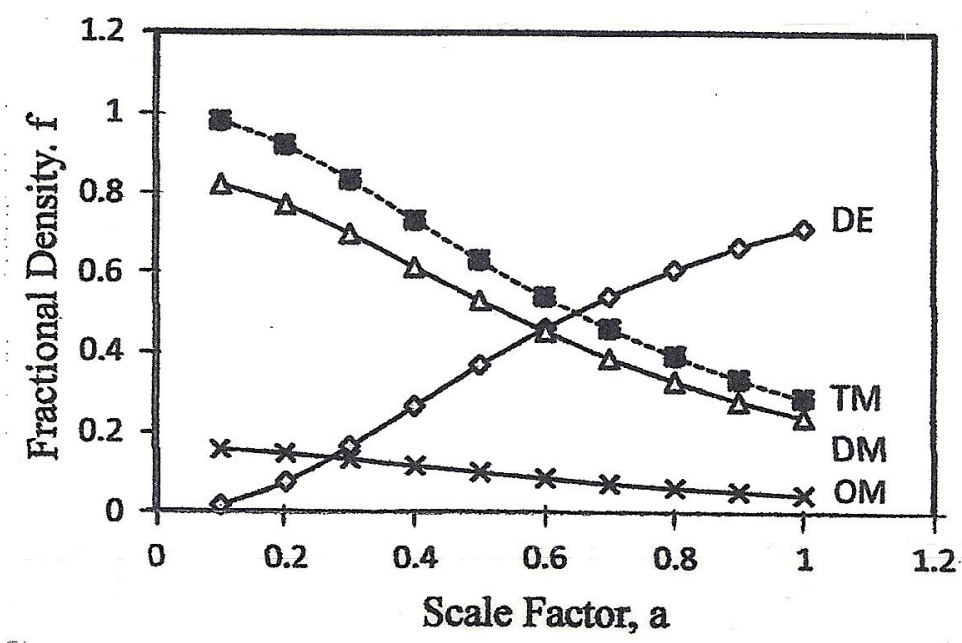

Fig.3- Fractional Energy Density (f) vs Scale Factor (a) DE- Dark Energy; DM-Dark Matter; OM-Ordinary Matter; TM- Total Matter

\subsection{NATURE AND PROPERTIES OF SPACE QUANTA}

Our quantum model of space provides a physical picture of space and match well published data on the composition of the universe at various epochs of evolution. The theory suggests some properties of space and space quanta:

1). They differ from electromagnetic radiation, hence cannot be detected by spectroscopic techniques. Space also differs from gravity. The space force exerts a pressure that counteracts the force of gravity; it is repulsive while the latter which is attractive.

2).They are associated with a scalar field rather than the vector field of electromagnetic waves. It might be that the space field is the "mother" field, from which arose both force fields and matter fields [13]. (Recall that at the "beginning", there were only energy and space from which were "born" radiation first and matter later).

3).The space waves (spaceons) are the carrier of energy in the universe. From this energy emanate radiation and all matter, as condensation products in the process of creation and evolution. The expansion of the universe is the expansion of space; the density of matter slows down the process and is accelerated by the dominance of dark energy. Space also probably acts as the container and reservoir of energy in the universe. Thus, virtual particles pop in and out of space; when matter and anti-matter annihilate and disappear, they go back to space.

4). Dark energy is pervasive and long range. We do not as yet know if there is a fundamental particle associated with the space field nor the equation that best describes its interactions.

\section{ON THE NATURE OF DARK MATTER}

Finally, we might say a few words about the other component of our the invisible universe, dark matter, since its known characteristics match well match well with a component present in our Phase Diagram (Fig.1). 
Dark matter [16] comprises $84 \%$ of the total mass of matter in the universe. At present, it makes up $24 \%$ of the total mass-energy of the universe It remains a mysterious, hypothetical form of matter.

Among properties that have been reported are:

1). it neither emits or absorbs electromagnetic radiation, hence it is difficult to study.

2). it moves without friction.

$3)$. it can only be detected through its gravitational effects on the motion of galaxies.

4). it is spread over large areas, like a cloud, and forms a "halo" around galaxies and clusters; its density decreasing as one moves away from the center [17].

5). it is also found in filaments between galaxies and clusters [18]. It has been observed that ordinary matter traces the path of dark matter; this has been attributed to a strong interaction between ordinary matter and dark matter.

We proceed with the premise that all components of universe formed during cosmic evolution and would have left their footprints in the sands of time, e.g, the CMB. The phase diagram (Fig.1) shows that the major phases in the formation of the universe are dark energy, ordinary matter (gasses, solids), and plasma. Any present-day component of the universe must originate from one of these phases. From the observed properties of dark matter, and known characteristics of plasma [19], it appears reasonable to make the assignment that dark matter corresponds to the plasma form of matter that existed during the photon epoch, just before recombination. The following facts support this contention:

1). WMAP data, mentioned earlier; show that dark matter constitutes the major component of the universe soon after the Big Bang. During this time, the universe consisted of a hot plasma of electrons, and ionized $\mathrm{H}$ and $\mathrm{He}$. It was opaque and cannot emit or absorb light. This state persisted until about 380,000 years after the Big Bang at which time recombination took place and the universe became transparent.

2). Other characteristics of plasmas match exactly those of dark matter. The plasma, like dark matter, hangs around like a cosmic fog around galaxies and clusters, making it invisible and difficult to characterize.

3). That ordinary matter traces the path of dark matter can be explained by the fact that upon recombination of electrons and positive ions in the dark matter plasma, ordinary matter is formed; the plasma evaporates into $\mathrm{H}$ and He gasses. Thus ordinary matter follows the trail of dark matter.

4). Filamentation is a characteristic of plasmas; they move without friction, since the ions do not have attractive interaction and move collectively instead [19]. This lack of interaction also explains the origin of dark matter halos [17] that hover around galaxies.

5). The dark matter plasma scatters elastically and hence do not clump or "stick together" thus remaining diffuse, fluffy, and"halo-like". Hence, galaxies cannot form directly from dark matter.

Moreover, plasma constitutes the major form of matter in the universe [19], most of it invisible. Dark matter being a plasma form of matter is a simpler rationale than an exotic particle whose existence has not been demonstrated. Dark matter should probably be called Plasma, so as to remove the mystery associated with its name.

\section{CONCLUSION}

A quantum model of space and a thermodynamic approach, appear to be useful constructs in providing a mechanism to help understand our dark universe. In our theory, dark energy is the 
energy of space and the component of the universe that causes its accelerating expansion. It is associated with a space force which emanate from a scalar field, dubbed as Quintessence. Our theory modifies present concepts of "empty space", "absolute vacuum" and the Cosmological Constant. Dark matter, on the other hand, is a plasma form of matter, similar to the state of the universe at the photon epoch, before recombination. It is the precursor in the formation of ordinary matter. Dark energy and dark matter are neither "dark" nor "mysterious", they are just invisible; one is transparent, while the other is opaque. Further work is necessary to better understand the nature and properties of space quanta, the space force, and its associated quantum field. Needless to say, our current hypotheses on these need further verification. The methods of Qunatum Field Theory could help achieve this [13]. Likewise, further work is needed to understand plasma in space (and dark matter halos), along with development of tools to carry them out.

\section{REFERENCES}

1. Frieman, J. A.; Turner, M. S.; Huterer, D. "Dark Energy and the Accelerating Universe". Annual Review of Astronomy and Astrophysics. 46 (1): 385-432 (2008)

2. Pain, R.; Astier, P. "Observational evidence of the accelerated expansion of the Universe". Comptes Rendus Physique. 13 (6): 521-538 (2012).

3. Futch, M., Leibniz, Metaphysics of Time and Space, Springer, NY (2008)

4. Jaffe, R.L, The Casimir Effect and the Quantum Vacuum. in: Physical Review D. 72, (2005)

5. Atkins, P. and De Paula, J, Physical Chemistry, W.H.Freeman, NY (2014)

6. Silk, J. The Big Bang, W.H. Freeman, N.Y. (1989)

7. Weinberg,S., The First Three Minutes, Basic Books, NY (1977)

8. Delsemme, A., Our Cosmic Origins, Cambridge University Press,UK (1998)

9. Bennett, C.L.; Larson, L.; Weiland, J.L.; Jarosk, N.; et al. "Nine-Year Wilkinson Microwave Anisotropy Probe (WMAP) Observations: Final Maps and Results". The Astrophysical Journal Supplement. 208 (2): 20 (2013)

10. Kirkwood, J. G., Oppenheim, I. Chemical Thermodynamics, McGraw-Hill, NY. (1961)

11. Stenger, V.J., The Comprehensible Cosmos, Prometheus Books, N.Y. (2006)

12. Weinberg, S., Cosmology, Oxford University Press, Oxford, UK (2014)

13. Zee, A., Quantum Field Theory in a Nutshell, $2^{\text {nd }}$ edition, Princeton Univ Press, NJ (2010).

14 Busca, N.G.; Delubac, T; Rich, J., et al, "Baryon Acoustic Oscillations in the Ly- $\alpha$ Forest of BOSS Quasars", Astronomy and Astrophysics, 552, A96 (2013)

15. Siegel, E., personal communication, August 2016

16. Trimble, V. (1987). "Existence and nature of dark matter in the universe". Annual Review of Astronomy and Astrophysics. Palo Alto, CA, Annual Reviews, Inc. 45: 425-472 (1987).

17. De Rujula, A., Jetzer, P., Masso, E., "On the Nature of the Dark Halo of Our Galaxy" , Astronomy and Astrophysics, 254, 99, (1992)

18. Dietrich, J., et al, "A filament of dark matter between two clusters of galaxies", Nature 487, 202-204 (2012).

19. Gurnett, D.A.; Bhattacharjee, A., Introduction to Plasma Physics: With Space and Laboratory Applications, Cambridge University Press, UK (2005) 